\title{
El parto en las variedades posteriores de vértice DESPRENDIMENTO ARTIFICIAL EN OCCIPITOSACRA
}

\author{
Tesis de Grado - Bogotá - 1948 \\ (RESUMEN)
}

\section{Docior HERNANDO NAVAS ANGEL}

\section{Generalidades.}

Después de estudiar las estadísticas de los servicios de Clínica Obstétrica cel Hospital de San Juan de Dios, comprendialas del $1^{\circ}$ de julio de 1947 al 30 de junio de 1948, de deducir de este estudio los cuadros estacísticos cule aparecen en primer capítulo de nujestro trabajo, ademiás de los índices, hemos llegado entre otras, a la conclusión de que el desprendimiento en variedad occípito-sacra de las variedades posteriores, no es una rareza clínica.

\section{Couscas.}

En opinión de los antiguos, los cartos en presentación de vértice cor desprendimiento en $O$. S., duraban mucho tiempo, eran causa de "malos dolores", en-una palabra, eran clasificados en la categoría de partos "coritra-natura". Mauriceau explicaba esta onomalía del parto de la cabeza diciendo: "Una de las causas particulares del trabajo prolongado en las variedades posteriores de vértice, es que el niño viene con la cara hacia alclanie y durante los dolores de ia madre, la musculatura abdominal comprime la matriz sobre las desigualdades de los miembros del feto. que están como la cara hacia adelante, lo que hace que la contracción, cousal del dolor, al ser interceptada. no pueda empujar tan fácilmente al niño hacia afuera, como lo haria si la compresión se hiciera sobre el dorso que es lo que ocurre cuando lo cara está hacia atrás, que es la situación natura?".

Demelin invoca como causa probable de desprendimiento en 0 . 5. una "oblicuidad anormal del útero" de donde resultaría una oblicuidad del tronco tetal con relación a la cabeza, a menos que ello no dependa de un utero con paredes flácidas, sobredistendido, como se observa en los emLarazos gemelares, los fibromas y los hidramnios.

Es lógico que el trabajo del parto. sea prolongado y el suirimiento fetal irecuente en las variedades posteriores, porque si las contracciones uterinas hacen progresar el parto cuando 
la cabeza fetal hace bloque con cl Mecanismo del parto de la cabeza. tronco, como sucede en las anteriores, en aquéllas, en cambio deflejan la cabeza que entonces recibe la fuerza solamente a través de la columna cervical que para el efecto es muy pequer̃a.

Devraigne opina que la $\mathrm{O}$. S. puede ser variedad primitiva en el encajamiento en las pelvis cifóticas, lo cue sería feliz eventualidad y que lo mismo puede suceder en los partos gemelares en que el segundo feto, peọueño, se encaja y desprende en $\mathrm{O}$. S. f'ero, salvo esta opinión que lue confirmada en nuestras observaciones, la O. S., es siempre secundaria y constituye una anomalía de la rotación interna del polo cefálico, y al respecto dice Demelin, que todas las variedades de posición en "las presentaciones de vértice, ya sean primitivamente posteriores, transversas 0 anteriores, pueden rotar, para desprenderse $a 0$.

S. y acusa de ello a la deflexión de la abeza.

Para enumerar entre 'as causas de artos en $\mathrm{O}$. S. tenemos tambien las normalidades de la pelvis, como el cesivo desarrollo de las espinas isnúticas, la morfología infundibuiirme, la estrechez transversa y las elvis planas que como se comprere. impiden la rotación y deflejan la rbeza.

Por parte del feto, son condiciones avorables a la no rotación y parto en o. S. la braquicefalia primitiva, la procidencia de un miembro y el asinclitismo persistente. En un plano de menor importancia, aunque a veces iuegan primordial popel, pueden considerarse el abdomen péndulo, las lesiones del segmento y del cuello, así como los tumores previos.

Normalmente, el vértice rota espontáneamente a O. P. El punto interesante del mecanismo es el tercer tiempo, porque en las variedades posteriores, del mismo modo que en las anteriores correspondientes, el oocipucio gira hacia adelante para colocarse bajo la síntisis púbica. Se pregunta, por qué esta evolución se hace hacia adolante y no hacia atrás. Son varios los autores que responden: 1 a) Baudelocque invoca como razón los planos inclinados de la pelvis, uno anterior, constituído por el agujero obturador y por el tallo óseo que se extiende de! isquión al estrecho superior; y el otro posterior, confundido con la gran escotadura ciática. Pero es difícil comfrender la manera como esos planos podrion dirigir el occipucio hacia adelante; b) Tyler Smith, cree que las espinas ciáticas obligan a la cabeza a rotar en tal o cual dirección, del mismo modo que la aguja de cambiós obliga a la locomotora a tomar tal $o$ cual vía. Pero la rotación del polo cefálico se hace, por lo común, cuando la parte más declive de la cabeza y las bosas parietales han franqueado el estrecho medio y por ende las espinas ciáticas. De esta suerte, dichos elementos óseos, no pueden jugar el presunto papel; c) Tarnier explica e! hecho con la teoría de la paianca, según la cual la parte posterior de la cabeza es menor en tamaño que el sincipucio. La frente encontraría pues un obstáculo relativamente más considerable por parte de la semicircunterencia anterior de la pelvis, que el sccipucio, y de ahí la rotación de este hacia adelonte; d) Dubois, en explica- 
ción un poco confusa, da a entender cue todo se debe a la acomodación, que parece ser la causa real de este movimiento de rotación. En efecto, Fuede afirmarse que el occipucio gira racia adelante, porque la cabeza colocada en O. P., se adapta mucho mejor al canal pélvico que en 0 . S. Basta comparar el perfil del ovoide cefálico con el de la pelvis naterna para darse cuenta de ello. Es natural, que siendo el lambda, la parte más declive en la presentación del vértíce. salga primero y para hacerlo debe seguir la curva pélvica y colocarse hacia adelante. Debe salir por el camino más corto, que es en este caso la pared vaginal anterior. La acomodación es pues, la cousa de la rotación a $\mathrm{O}$. P.

Irregularidades del mecanismo en las variedades posteriores.

Pueden presentarse en cada uno de los tiempos del partc.

Io a) Defecto en la disminución del tamaño de la presentación por dificul. tad en el acaballamiento óseo debido a osificación muy avanzada.

b) Flexión insuficiente de ia cxbeza $r$ a veces conversión en presentaciowes de frente o cara.

2. Rotación internx. El movimiento de rotación puede hacerse demasiado pronto o demasiado tarde como cuanà el occipucio gira hacia delante, sólo al llegar a las vecindades de la vulva. Este retardo es frecuente, cuando el leto es pequeño o la pelvis muy amplia y en este caso pierde la teoría de la acomodación parte de su validez. Asímismo, la rotación interna es susceptible de irregularidrades por exceso o por defecto, según cue el occipucio no alcance a llegar o la sínfisis púbica o vaya más allá de ella.

Pero la principal anomalía de este tiempo de parto es la rotación del occipucio hacia atrás. Se realiza de dos differentes maneras:

Siendo antero-posterior la sutura sagital, estando la fontanela menor hacia atrás y el bregma más o menoz cculto detrás de la sínfisis pubiana, el occipucio se hunde en el periné, lo cistiende y luégo de un gran trabajo y de gran flexión de la cabeza, emerse fuera de la horquilla. El suboccipucio se apoya entonces sobre ella y extendiéndose el vértice se ve aparecer sucesivamente el bregma, la frerte, la nariz, la boca y el mentón, completándose asi el parto de la cabeza y en la mayoría de las veces produciéndose un gran desgarro perineal.

$O$ bien, con mecanismo diferente, la cabeza se defleja, el occipucio distiende la máximun, la parte posterior del periné, aparece primero el bregma, luégo la trente y en este estado se detiene la extensión cefálica. Esta forma de desprendimiento hizo decir a Auvard, que muchas occípito-sacras no son más que frentes ignoradas.

\section{TRABAJO}

Siendo nuestro interés primordial al realizar este trabajo, demostrar en in posible por medio de estudios estadísticos, la mayor incidencia del trabajo prolongado y del sufrimiento fetal en las variedades posteriores entre las presentaciones del vértice, hemos elaborado unos cuadros estadísticas y deducido de ellos 
muy valiosos datos. En el original de nuestro trabajo aparecen dichos cuadros.

Fueron objeto de nuestro estudio 5.093 historias clínicas, entre las que encontramos solamente 129 casos de variedades posteriores, que son las cue integran nuestros cuadros esicdísticos. Pero es claro que no fueron solamente estos los casos que debieron presentarse. El hecho es que $n o$ en todas las historias clínicas se encuentra anotada la variedad de posición en el encajamiento, porque no en todas las pacientes se practica el tacto, por innecesario, o porque debido a causas especiales no se ha podido determinar la variedad de posición. De esta suerte, los índices y porcentajes deducidos no son ni pueden er absolutos, pero sí muy elocuentes or cuanto sirven de base para conceptos que expresamos.

Entre las 5.093 historias clínicas esdiadạs, encontramos descripciones de 291 aplicaciones de fórceps, en loda clase de variedades de posición.

Los datos suministrados por los 129 asos de variedades posteriores de zestros cuadros, son:

\section{Primíparas: $65,11 \%$}

Multiparas: $34,89 \%$

Promedio de edad materna: 24 años

Encaiamientos en OIDP: $80,20 \%$.

Encajamientos en OIIP: $18,60 \%$.

Encajamientos primitivos en 0 . S.: $:, 20 \%$.

Promedio de duración del trabajo: 51 horas 40 minutos (!).

Casos de sufrimiento fetal: $45,73 \%$. Mortalidad infantil y mortinatalidad debidas al sufrimiento fetal: $16,27 \%$
Fórceps en variedades posteriores: $51,93 \%$.

Desprendimientos en O. P.: $88,38 \%$.

Desprendimientos en 0 . S.: $11,62 \%$. Desgarros perineales: $20,93 \%$

Episiotomías: $24,03 \%$.

Perinés ilesos: $55,04 \%$.

Sorprende al ánimo más desprevenido, el resultado alarmante, por los

- datos que suministra, de este estudio estadístico.

¿A qué es debida esta excesiva diración del trabajo y sus peligrosas consecuencias? Sobre todo a la dilatación lenta del cuello, sobre el cual la cabeza se apoya mal así como a la flexión insuficiente. Esto explica que a pesar de una dilatación completo, la mujer se esfuerza en vano por expulsar, llevando mal las contracciones sobre la cabeza deflejada. Después, cuando ésta llega al piso perineal, si se flexiona, podrá salir, después de haberlo distendido y de provocar el desgarro. Deflejada, también podrś desprenderse espontúneamente, pero este desprendimiento será largo y pe. noso.

\section{Diagnóstico.}

Es trecuentemente desconocido, poroue no se piensa suficientemerte en la $O$. S. Es bien sabido, que la mayoría de las occípito-posteriores rotan normalmente $\alpha$ O.P. si están flexionadas o si lo son artificialmente. Pero aparte de que estas variedades rotan a veces $a O$. S., las anteriores y transversas también pueden hacerlo en de. terminadas circunstancias de defiexión cefálica. 
Fara el médico no especializado, cada vez que la sutura sagital es antero-posterior, la rotación interna está hecho; se trata seguramente de una O. P. y todo va a marchar bien; con algunas buenas contracciones $e l$ parto va a verificarse y se incita a la parturienta a pujar enérgicamente. Al cabo de una prolongada espera, anle la evidencia de algo anormal, se hace un nuevo tacto vaginal y si no lo inpide una gran bosa sero-sanguínea, se encuentra la sutura sagital y hacia adelante, por debajo de la síntisis pubiana, la ionianela bregmática. Se r.ace entonces el diagnóstico de $\mathrm{O}$. S. y se halla la razón de la distocia.

\section{Pronóstico.}

Varía, según que el diagnóstico sea o no, hecho con oportunidad. En el caso positivo, con conductas determinadas, se podrá resolver ei problema. Il feto está más amenazado en las O. S., que en las O. P. porque el trabajo es más largo.

\section{Conductas.}

a) Espera. La técnica obstética moderna tiende a la prevención de lo.s accidentes maternos y fetales. Si bien es cierto que todas las presentacione; de vértice rotan fisiológicamente para desprenderse, a O. F., las variedades posteriores con alguna frecuencia y por razones obvias, se hacen distócicas. Por esto, la exageración de la "inteligente espera", nos parece una conducta peligrosa. El trabajo en las variedades posteriores se prolonga, la paciente se extenúa, el suirmiento fetal se hace notorio y casi siemore si llega a la necesidad de intervenir en condiciones adversas.

b) Maniobra digitales. Ante el chadro distócico de una variedad poste1ior de vértice que no sigue la evolición fisiológica, surge en primer luger Ia indicacion de hacer la rotacien con maniobras manuales, como la mznicbra oe Hodge, que trata de aumentar la llexión de la cabeza por medio ie presiones digitales ejercidas sobre el sincipucio, durante las contracciones uterinas y de hacer luégo la rolación manual para lo cual debe operar la mano profundamente introducida en la cavidad uterina. Entre otras razores, aducimos el grave peligro de infección del endometrio para justificar nuestro rechazo de esta conducta, demorada en su ejecución y fácilmenie ieemplazable por otras más técnicas

c) Ocitócicos. Entre nosotros el Profesor Ramírez Merchán, ha sintetizado en uno de los apartes de su trabajo de agregación titulado Conductas obstátricoxs controvertioles, lo que actucimente puede y debe saberse sobre ccitócicos, y refiriéndose a su uso en los variedades posteriores de vértice, se expresa asi: "Se nos ocurre, que : hoy especialistas que proponen abandonar en totalidad el uso de la pituitrina, no adhiriendo nosotros a tal concepto, si preconizamos relegatla de manera absoluta en las preseniaciones de cara de frente y aún en las de vértice atascadas en O. D. P.". Por nuestra parte, atrevidamente diríamcs cue las variedades posteriores de vértice podrían considerarse para el eferto, como colocadas en el "límite de la distocia". 
d) Fórceps. ¿Con qué objeto convertirse en espectador de los padecimientos de la madre en trabajo con una variedad posterior de vértice que no rota, o verificar rotaciones manuales de éxito dudoso si llenadas las indicaciones podemos verificarlas en sentido anterior o posterior y en más corto tiempo con el fórceps? Para hacerlo existen varias técnicas, enumeradas y descritas por el Profesor Ramírez Merchán en su Presentación de una técnica original para las aplicaciones del Fórceps en las variedades posteriores del vértice, publicada on la Revista Colombiana de Obstetricia y Ginecología, número 2, volumen 1?, a saber: Técnica Clásica, Técnica de Scanzoni, Técnica Invertida, Maniobra le De Lee (The Key in lock), Técnica e Tatelbaum desprendimiento en -licua, Técnica Original, presentada or el Protesor Ramírez y por último. tación y desprendimiento en $\mathrm{O}$. S. bjeto principal de nuestro trabajo.

De todas estas técnicas, la más coccida y usada es la clásica, con roucion a O. P. Requiere cuatro tiems a saber: flexión, correción del asinnemo, rotación y extracción las dos meras se logran con las maniobras muales de que hablamos anteriornte. Después de esto, se colocan ramas del fórceps en el diámetro hicuo opuesto al que sigue la sutura sagital. Viene luégo la rotación Q ve para ser correcta debe practicaren forma progresiva por medio de modificación de De Lee (la llave en cerradura), que traía de imitar el oceso natural, imprimiendo a la caeza un "movimiento de serpenteo". os o más tomas son necesarias para hacer esta rotación de $135^{\circ}$, par r lleaar a la extracción con la curva pelvica del fórceps orientada hacia ade. lante. Fero puede correrse la coningencia de que esta rotación se haga difícil o imposible, impidiendo al cirujano ceñirse a la regla clásica del desprendimiento en O. P. y por otra parte esta operación requiere bastante tiempo para ser suave y con todo, a pesar de la relativa inocuidad del torceps, no dejan de presentarse desgarros en las paredes vaginales, Es entonces cuando surge la necesiàad de utilizar otra técnica para terminar el parto.

e) Operación cesárex. No está precisamente entre las indicaciones de la cesárea la variedad posterior del vértice, pero en cambio sí está plenamente indicada en muchas de los casos que constituyen la etiologio de esa variedad de posición. En éfecto, pel vis infundibuliformes, pelvis transversalmente estrechas, pelvis planas, indican una vez diagnosticadas, la thtervención por vía alta. Lo mismo podría decirse de los grandes edemas del cuello, ton frecuentes en esta modalidad del parto.

Ante los alormantes datos suminis. trados por nuestro estudio estadístico de las variedades posteriores, creet mos justificada la preconización, al lado de las otras conductas conocidas, diferentes de la clásica, entre las que brilla con luz propia la original presentada por el Frofesor Ramírez Merchán, de la técnica de la rotación y desprendimiento en $\mathrm{O}$. S. No pretendemos ser originales, ni buscar aceptación general. Nuestro único anhelo es cont 
tribuír al estudio de uno de los probiemas de la especialidad.

Muy pocas veces se realiza espontáneamente la rotación hacia atrás y el desprendimiento en $\mathrm{O}$. S. Afortunadamente, porque muy a menudo esto no se ha previsto. Pero, si ello es posible por los medios naturales, ¿por que no ha de serlo por los instrumentales?

Habiendo preconcebido la idea de intentar siempre en los casos de variedades posteriores la rotacion posterior antes de intentarla hacia el pubis, lo hicimos así en todos los casos en que había completa indicación de fórceps, y eh ninguno de ellos encontramos dificultad en las maniobras de rotación y extracción. Así pues, es esta la variación que proponemos, de escasa importancia si la valoramos por las dificultades aue ocasionalmente pueda acarrear, pero de gran utilidad si se miden sus ventajas de rapidez del acto operatorio, de inocuidad para el feto y aún para la madre.

En la rotación en sí, raro será encontrar dificultades, pero si eso ocurriera nada hay en e! intento fallido que impida seguir entonce; cualquierci de las otras técnicas ya enumeraàs. En donde sí puede haber dificultades y peligros, especialmente para la integridad perineal de la madre, es en las maniobras de desprendimiento, pero esos peligros pueden conjurarse fúcilmente procurando en primer lugar reducir la deflexión de la cabeza, tántas veces acusada de ser la causa de la distocia en estos casos. Para lograrlo basta, una vez cumplida la rotación, teniendo una toma biparietal, directa para la madre, e invertida para el teto, hacer tracciones hacia ade- lante hasta que la presentación haya franqueado el estrecho medio; cuando el parto esté en este punto $y$ se inicien las tracciones para el desprendimiento, éstas deben hacerse hacia adelante y arriba con el objeto de llevar ia espina nasal del frontal a colocarse frente al borde inferior de la sinfisis pubiana matema. Así ésta servirá de punto de apoyo para desprender el occipucio con tracciones que se hacen nuevamente hacia adelante. Se retiran entonces las cucharas del fórceps y si las condiciones del periné lo indican, se practica episiotomía paramediana, que tiene la ventaja de que si no resultare suficientemente amplia y se produjere un desgarro sobreagregado, éste seguirá la línea trazada por aquélla y por lo tanto no comprometerá la integridad del estinter anal. Siguiendo esta técnica especial que nos flie serialada y dictada por los Profesores José del C. Acosta, Carlos J. Mojica Y Rafael F. Ramírez M., no hemos encontrado dificultades en ninguno de los casos tratados, cuyas historias clínicas aparecen en el capítulo correspondiente de nuestro trabajo.

\section{Conclusiones.}

12. En todas las ocasiones en que el trabajo del parto en presentación de vértice se prolongue, especialmente en el período de descenso o en el de desprendimiento, sin causa aparente que jo justifique, debe pensarse en una variedad posterior que no ha podido rotar, que trata de hacerlo o lo ha hecho a occípito-sacra.

2a El parto en las variedades occípito-posteriores, con desprendimients 
en U. 2., es en ucusiuites velthindi. das, tan fácil y eutócico como cuando termina en $O$. P.

3: La causa principal de largo trabajo en las occípito-posteriores, es la dellexión de la cabeza.

4 a El desprendimiento en $O$. S., no es una rareza clínica.

$5^{2}$ Las occípito-posteriores pueden considerarse en el "límite de la distocia".

6? Se justifica plenamente la intervención instrumental en estos casos, sin esperar las complicaciones de tan irecuente ocurrencia.

$7^{2}$ Al aplicar el fórceps en estos casos, se puede intentar la rotación de la variedad posterior a $\mathrm{O}$. S. Y desprender en esta variedad, con las moditicaciones de técnica descritas. Si no es posible hacerlo y sin necesidad de cambiar de toma, debe entonces seguirse la conducta clásica u otra de las ya enumeradas, rotando a $O$. $P$. La extracción en $O$. S. tiene las ventajas de abreviar la intervención, ser rnenos traumática parri la madre y el feto, no requerir sino una sola toma on el fórceps, una rotación mínima de 5 grados y ser casi tan fácil como la echa en O. P.

8: El desgarro perineal anunciado for la mayoría de los autores para estos casos, no siempre se produce, y puede evitarse con la episiotomía cportuna que siendo paramediana trazará el camino al desgarro, si io hay, $\sin$ que comprometa el esfinter anal.
1-Acosta S. Hernando. Conterencias de Obstetricia. 1945. Bogotá.

2-Peralta C. Rafael. Conferencias de Obstetricia. 1946. Bogotá.

3-Ramírez Merchán Raíael F. "Conductas obstétricas controvertibles". Revista de la Facultad de Medicina. Números 7 y 8. 1948. Bogotá. 4-Martínez Oscar. "Algunos aspectos del parto en las primiparas". Tesis de grado. Bogotá. 1947.

5-Pérez Mánuel Luis. Tratado de Obstetricia. .

6-Moragues Bernat Joime. Clínico Obstétrica.

7-De Lee-Greenhill. Principles and Practice of Obs'etrics.

8-Fabre. Manual d'Obstetrique.

9-Devraigne L. Manua! d'Obstetrique.

10-Recassens Girol Traité d'Obstetrique..

11-Auvard. Traité practique d'Aco aruchements.

12-Rouvier Jules M. Traité practique d'Obstetrique normale

13-Bumm Emest. Precís d'Obstetrique,

14-Farabeuf L. H. et Vornier Henri. Introduction $\propto$ l' etude clinique et a la practique des accouchements. 15-Devraigne L. "Les occipito-sacreés". Le Monde Medical. Mayo 1946. 